\title{
Transmitted field in the lossy ground from ground penetrating radar (GPR) dipole antenna
}

\author{
D. Poljak \& V. Dorić \\ University of Split, Croatia
}

\begin{abstract}
The paper deals with the evaluation of transmitted electric field in the ground due to the GPR dipole antenna. The frequency domain formulation is based on the integro-differential equation of the Pocklington type. The influence of the earthair interface is taken into account via the simplified reflection/transmission coefficient arising from the Modified Image Theory (MIT). The space-frequency Pocklington equation is solved via the Galerkin-Bubnov variant of the Indirect Boundary Element Method (GB-IBEM) and the corresponding transmitted field is obtained by numerically computing field integrals. Some preliminary results for the electric field transmitted into material media are presented.

Keywords: ground penetrating radar, dipole antenna, lossy half-space, transmitted field, numerical solution procedures.
\end{abstract}

\section{Introduction}

Ground-penetrating radar (GPR) finds numerous applications in different areas of geophysics and underground engineering, such as civil engineering [1]. An important, one of the most critical, component regarding GPR system performance is an antenna whose type and size strongly depend on particular application [2]. Namely, the knowledge of the energy transmitted into the dissipative half-space enables better antenna design and more realistic interpretation of the target reflected wave.

The use of physically larger, low frequency antenna provides deeper penetration into the ground, while high frequency antennas are convenient for the tasks requiring less penetration depth and better resolution. 
Consequently, a deeper insight into the behavior of the field transmitted within the lossy ground is of continuous interest in GPR research. In general, the analysis can be carried out in the frequency or time domain, respectively, [3-5]. Many efficient GPR antenna models based on the Finite Difference Time Domain (FDTD) method of solution have been reported, e.g. [6, 7].

Contrary to the widely used FDTD approach the present paper deals with the assessment of transmitted electric field in the ground due to the GPR dipole antenna by means of the Boundary Element Method (BEM). The formulation is based on the space-frequency integro-differential equation of the Pocklington type and corresponding filed formulas. The presence of the earth-air interface is taken into account via the simplified reflection/transmission coefficient arising from the Modified Image Theory (MIT). The space-frequency Pocklington equation is numerically solved via the Galerkin-Bubnov variant of the Indirect Boundary Element Method (GB-IBEM) and the corresponding transmitted field is obtained by numerically computing the related field integrals. Some illustrative results for the transmitted electric field are given in the paper.

\section{Formulation}

Geometry of interest is related to the dipole radiating above a lossy medium, as it is shown in Fig 1.

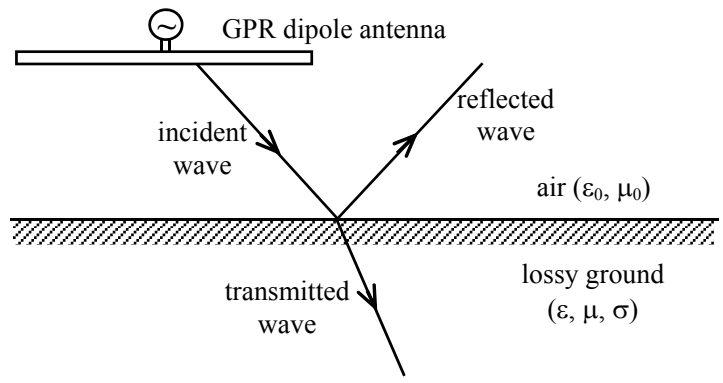

Figure 1: GPR dipole antenna above a lossy half-space.

Generally, a directive transmission of signal into a material half-space can be analyzed by means of the rigorous Sommerfeld integral formulation, or the approximate Fresnel reflection/transmission coefficient capproach [3]. The validity of each approach has been discussed elsewhere and some general remarks can be, for example, found in [8] or [9]. This work features the use of the modified image theory (MIT) [9] in the transmitted field formula.

The integro-differential equation for the current induced along the dipole can be derived by enforcing the interface conditions for the tangential components of the electric field at the wire surface:

$$
\vec{e}_{x} \cdot\left(\vec{E}^{e x c}+\vec{E}^{s c t}\right)=0
$$


where the excitation field is composed from the incident field $\vec{E}^{\text {inc }}$ and the field reflected from the lossy ground $\vec{E}^{r e f}$ :

$$
\vec{E}^{e x c}=\vec{E}^{i n c}+\vec{E}^{r e f}
$$

The scattered field is expressed in terms of magnetic vector potential $\vec{A}$ and electric scalar potential $\phi$ :

$$
\vec{E}^{s c t}=-j \omega \vec{A}-\nabla \phi
$$

According to the thin wire approximation [3] expression (3) becomes:

$$
E_{x}^{s c t}=-j \omega A_{x}-\frac{\partial \phi}{\partial x}
$$

with:

$$
\begin{gathered}
A_{x}=\frac{\mu}{4 \pi} \int_{-L / 2}^{L / 2} I\left(x^{\prime}\right) g\left(x, x^{\prime}\right) d x^{\prime} \\
\varphi(x)=-\frac{1}{j 4 \pi \omega \varepsilon_{0}} \int_{-L / 2}^{L / 2} \frac{\partial I\left(x^{\prime}\right)}{\partial x^{\prime}} g\left(x, x^{\prime}\right) d x^{\prime}
\end{gathered}
$$

where $I\left(x^{\prime}\right)$ is the induced current along the antenna, while $g\left(x, x^{\prime}\right)$ denotes the total Green function:

$$
g\left(x, x^{\prime}\right)=g_{0}\left(x, x^{\prime}\right)-R_{T M} g_{i}\left(x, x^{\prime}\right)
$$

where $g_{0}\left(x, x^{\prime}\right)$ is the free space-Green function:

$$
g_{o}\left(x, x^{\prime}\right)=\frac{e^{-j k_{o} R_{o}}}{R_{o}}
$$

while $g_{\mathrm{i}}\left(x, x^{\prime}\right)$ arises from the image theory:

$$
g_{i}\left(x, x^{\prime}\right)=\frac{e^{-j k_{o} R_{i}}}{R_{i}}
$$

where $R_{o}$ and $R_{i}$ denote the corresponding distance from the source to the observation point, respectively.

The reflection coefficient for the transverse magnetic polarization $R_{\mathrm{TM}}$, which accounts for the presence of a lower lossy medium, is given by:

$$
R_{T M}=\frac{n \cos \Theta-\sqrt{n-\sin ^{2} \Theta}}{n \cos \Theta+\sqrt{n-\sin ^{2} \Theta}}
$$

The refraction index $n$ and angle $\theta$ are given by:

$$
n=\varepsilon_{r}-j \frac{\sigma}{\omega \varepsilon_{0}}, \Theta=\operatorname{arctg} \frac{\left|x-x^{\prime}\right|}{2 h}
$$

Inserting (4)-(6) into (2) yields the Pocklington's integro-differential equation for the unknown current distribution induced along the dipole: 


$$
E_{x}^{e x c}=j \omega \frac{\mu}{4 \pi} \int_{-L / 2}^{L / 2} I\left(x^{\prime}\right) g\left(x, x^{\prime}\right) d x^{\prime}-\frac{1}{j 4 \pi \omega \varepsilon_{0}} \frac{\partial}{\partial x} \int_{-L / 2}^{L / 2} \frac{\partial I\left(x^{\prime}\right)}{\partial x^{\prime}} g\left(x, x^{\prime}\right) d x^{\prime}
$$

The transmitted electric field components in the $\mathrm{XZ}$ plane are given, as follows:

$$
\begin{gathered}
E_{x}=\frac{1}{j 4 \pi \omega \varepsilon_{e f f}}\left[-\int_{-L / 2}^{L / 2} \frac{\partial I\left(x^{\prime}\right)}{\partial x^{\prime}} \frac{\partial G\left(x, x^{\prime}, z\right)}{\partial x^{\prime}} d x^{\prime}-\gamma^{2} \int_{-L / 2}^{L / 2} G\left(x, x^{\prime}, z\right) I\left(x^{\prime}\right) d x^{\prime}\right] \\
E_{z}=\frac{1}{j 4 \pi \omega \varepsilon_{\text {eff }}} \int_{-L / 2}^{L / 2} \frac{\partial I\left(x^{\prime}\right)}{\partial x^{\prime}} \frac{\partial G\left(x, x^{\prime}, z\right)}{\partial z} d x^{\prime}
\end{gathered}
$$

where $G\left(x, x^{\prime}\right)$ :

$$
G\left(x, x^{\prime}\right)=\Gamma_{t r}^{M I T} g_{E}\left(x, x^{\prime}, z\right)
$$

And the transmission coefficient $\Gamma_{t r}^{M I T}$ arising from the modified image theory (MIT) is given by:

$$
\Gamma_{t r}^{M I T}=\frac{2 n}{n+1}
$$

The solution of integro-differential equation (12) can be obtained by applying the Galerkin-Bubnov scheme of the Indirect Boundary Element Method (GBIBEM). Once calculating the induced current the related transmitted electric field is obtained by numerically computing field integrals (13) and (14).

\section{Numerical procedures}

The Galerkin-Bubnov scheme of the Indirect Boundary Element Method (GBIBEM) for the solution of integro-differential equation (12) is documented in detail elsewhere, e.g. in [3]. The components of the electric field transmitted into the material medium due to the dipole radiation are evaluated using the BEM formalism. The procedure is outlined in this section, for the sake of completeness.

The current and its first derivative at the $i$-th boundary element are given by:

$$
\begin{gathered}
I\left(x^{\prime}\right)=I_{1 i} \frac{x_{2 i}-x^{\prime}}{\Delta x}+I_{2 i} \frac{x^{\prime}-x_{1 i}}{\Delta x} \\
\frac{\partial I\left(x^{\prime}\right)}{\partial x^{\prime}}=\frac{I_{2 i}-I_{1 i}}{\Delta x}
\end{gathered}
$$

where $I_{1 i}$ and $I_{2 i}$ are the values of current at the local nodes of the $i$-th boundary element, with coordinates $x_{1 i}$ and $x_{2 i}, \Delta x=x_{2 i}-x_{1 i}$ denotes the element length.

Discretizing (13) and (14) and substituting (17) and (18) into (13) and (14) results in the following expressions:

$$
\begin{gathered}
E_{x}=\frac{1}{j 4 \pi \omega \varepsilon_{e f f}} \sum_{i=1}^{N_{j}}\left[-\frac{I_{2 i}-I_{1 i}}{\Delta x_{j}} \int_{x_{1 i j}}^{x_{2 i}} \frac{\partial G\left(x, x^{\prime}, z\right)}{\partial x^{\prime}} d x^{\prime}-\gamma^{2} \int_{x_{1 i}}^{x_{2 i}}\left[I_{1 i} \frac{x_{2 i}-x^{\prime}}{\Delta x}+I_{2 i} \frac{x^{\prime}-x_{1 i}}{\Delta x}\right] G\left(x, x^{\prime}, z\right) I\left(x^{\prime}\right) d x^{\prime}\right] \\
E_{z}=\frac{1}{j 4 \pi \omega \varepsilon_{e f f}} \sum_{j=1}^{M} \sum_{i=1}^{N_{j}} \frac{I_{2 i j}-I_{1 i j}}{\Delta x_{j}} \int_{x_{1 i j}}^{x_{2 i j}} \frac{\partial G\left(x, x^{\prime}, z\right)}{\partial z} d x^{\prime}
\end{gathered}
$$

where $N_{j}$ denotes the total number of boundary elements along the wire. 
Integrals in (19) and (20) are numerically evaluated using the Gaussian quadrature. The quasi-singularity of the Green function is avoided by approximating the first-order differential operator with finite differences [3].

\section{Numerical results}

The computational example is related to the dipole antenna of length $L=1 \mathrm{~m}$ and radius $a=2 \mathrm{~mm}$ horizontally placed at height $h=0.25 \mathrm{~m}$ above a real ground with permittivity $\varepsilon_{\mathrm{rg}}=10$ and conductivity $\sigma=10 \mathrm{mS} / \mathrm{m}$. Terminal voltage is $V_{\mathrm{T}}=1 \mathrm{~V}$. The operating frequency is varied from $1 \mathrm{MHz}$ to $300 \mathrm{MHz}$.

Figs 2 to 4 show the related fields components for $f=1 \mathrm{MHz}, f=10 \mathrm{MHz}$ and $f=100 \mathrm{MHz}$.

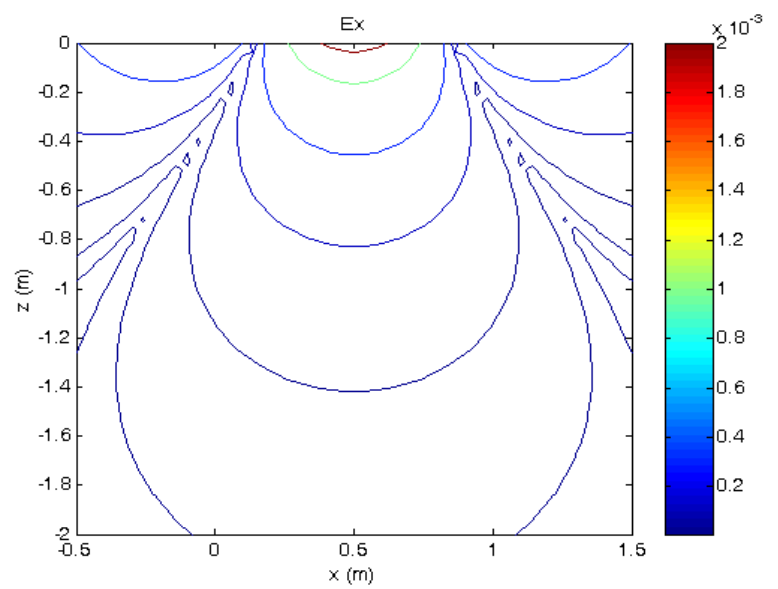

(a) $E_{\mathrm{x}}-$ component.

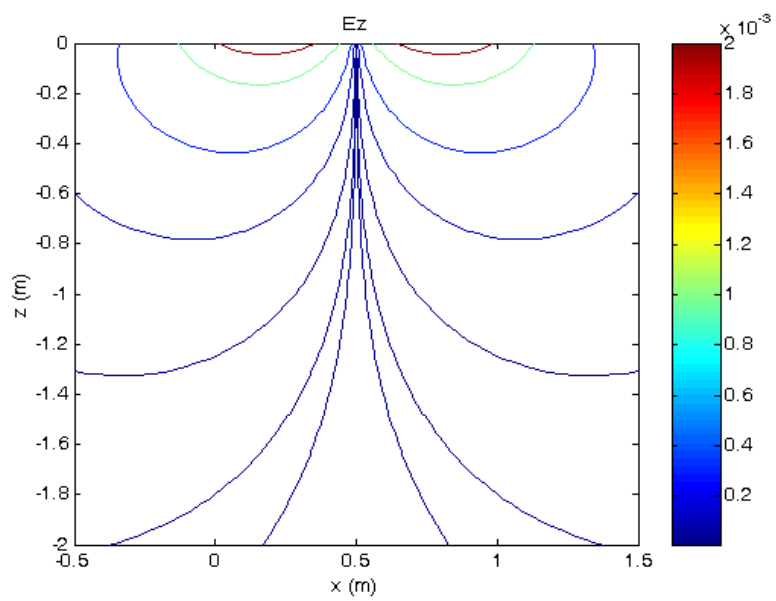

(b) $E_{\mathrm{z}}$ - component.

Figure 2: $\quad$ Transmitted field $(\mathrm{V} / \mathrm{m})$ into the ground at $f=1 \mathrm{MHz}$. 


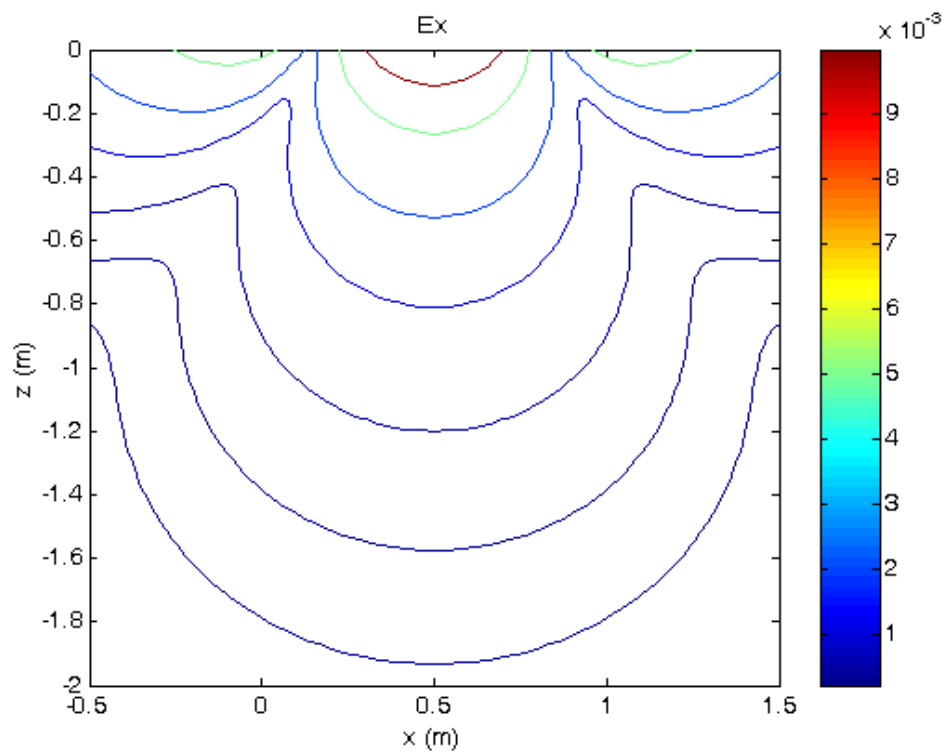

(a) $E_{\mathrm{x}}-$ component.

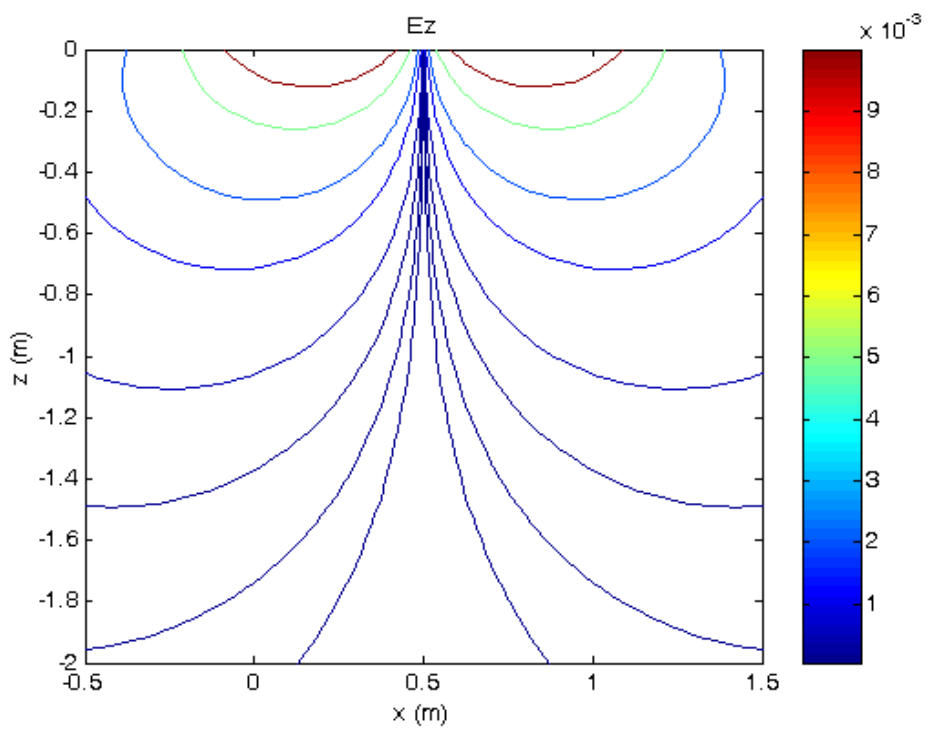

(b) $E_{\mathrm{z}}-$ component.

Figure 3: Transmitted field $(\mathrm{V} / \mathrm{m})$ into the ground at $f=10 \mathrm{MHz}$. 


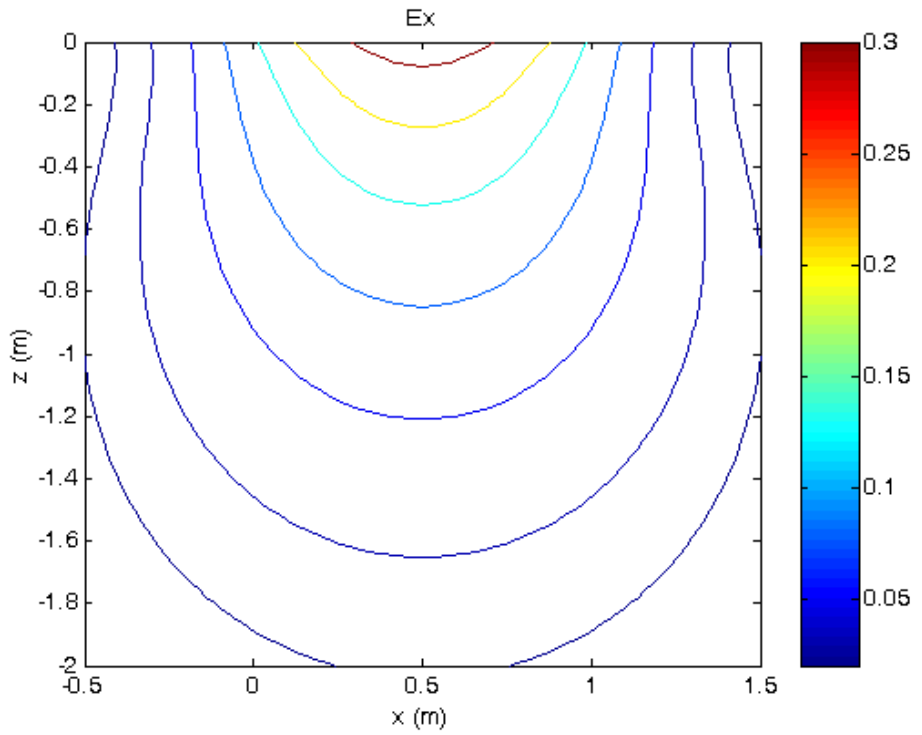

(a) $E_{x}$ - component.

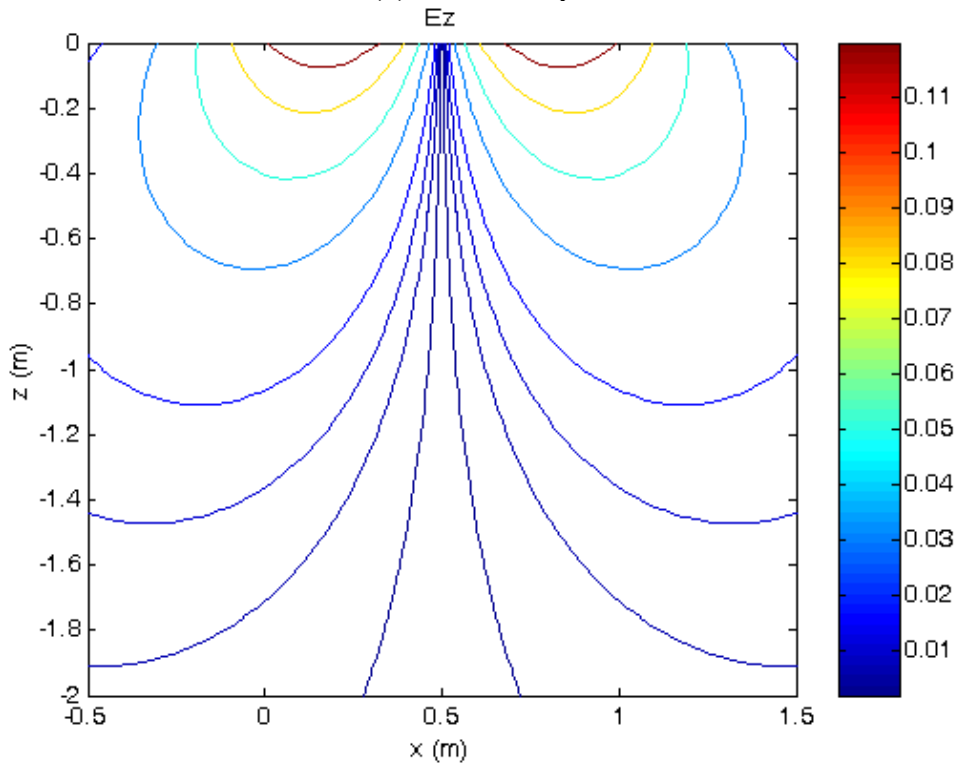

(b) $E_{\mathrm{z}}$ - component.

Figure 4: Transmitted field $(\mathrm{V} / \mathrm{m})$ into the ground at $f=100 \mathrm{MHz}$.

Analyzing the numerical results presented in Figs 2 to 4 it can be observed that the field distribution remains relatively stable over the considered frequencies.

Fig. 5 shows the $E_{\mathrm{x}}$ component of the transmitted field versus depth in the broadside direction for different operating frequencies. 


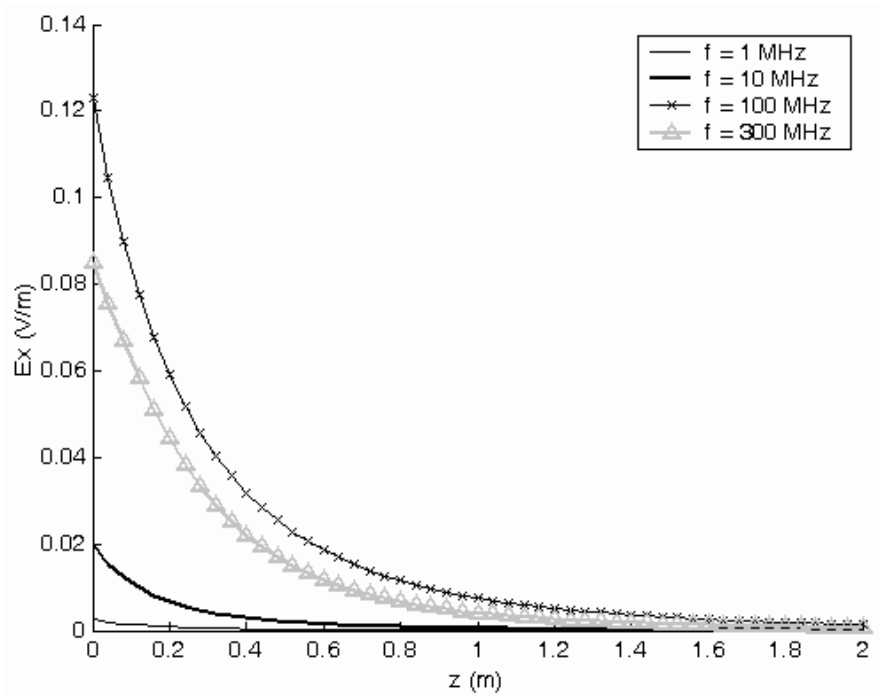

Figure 5: Broadside transmitted field $(\mathrm{V} / \mathrm{m})$ into the ground for different frequencies.

Note that, due to the symmetry of the problem, $E_{\mathrm{z}}$ component in the broadside direction is zero. This is easily visible in Figs $2 b, 3 b$ and $4 b$.

\section{Conclusion}

The paper deals with the analysis of the electric field transmitted into the material half-space due to the GPR dipole antenna radiation. The frequency domain formulation is based on the Pocklington integro-differential equation and related field formulas. The influence of the earth-air interface is taken into account via the simplified reflection/transmission coefficient arising from the Modified Image Theory (MIT). The Pocklington equation is numerically solved via the GalerkinBubnov variant of the Indirect Boundary Element Method (GB-IBEM) and the corresponding transmitted field is determined by using BEM formalism, as well. Some computational examples for the electric field transmitted into the material medium are presented. This work should be considered as an opener to the subject and the future work will deal with coupled dipole arrays above a lossy ground for GPR applications in both frequency and time domain. Moreover, within future activities it is planned to carry out a stochastic collocation analysis of the transient current induced along the wires radiating over a lossy medium.

\section{Acknowledgement}

This work benefited from networking activities carried out within the EU funded COST Action TU1208 “Civil Engineering Applications of Ground Penetrating Radar". 


\section{References}

[1] L. Pajewski et al., Applications of Ground Penetrating Radar in Civil Engineering - COST Action TU1208, 2013.

[2] C. Warren et al., Radiation Characteristics of a High-Frequency Antenna in Different Dielectric Environments, $15^{\text {th }}$ International Conference on Ground Penetrating Radar - GPR 2014, pp. 796-801, Brussels, Belgium, 2014.

[3] D. Poljak, Advanced Modeling in Computational electromagnetic Compatibility, John Wiley and Sons, New York 2007.

[4] D. Poljak et al., Transient Analysis of Two Coupled Horizontal wires over a Real Ground, IEE Proc. Microwave Antennas Propagat., 147, 87-94, 2000.

[5] M. Fernandez Pantoja et al., Time Domain Analysis of Thin Wire Antennas over Lossy Ground using the Reflection-Coefficient Approximation, Radio Science, Vol. 44, 2009.

[6] J. M. Bourgeois, G. Smith, A Fully Three- Dimensional Simulation of a Ground-Penetrating Radar: FDTD Theory Compared with Experiment, IEEE Transactions on Geoscience and Remote Sensing, Vol. 34, No 1, Jan., pp. 361996.

[7] L. Gürel, U. Oguz, Three-Dimensional FDTD Modeling of a Groundpenetrating Radar, IEEE Trans. On Geoscience and remote Sensing, Vol. 38, No 4, July 2000.

[8] E. K. Miller, A. J. Poggio, G. J. Burke, E. S. Selden, Analysis of wire antennas in the presence of a conducting half-space. Part II. The horizontal antenna in free space, Canadian Journal of Physics, 50, 1972, pp. 2614-2627.

[9] D. Poljak, Poljak, K. El Khamlichi Drissi, K. Kerroum, S. Sesnic, Comparison of analytical and boundary element modeling of electromagnetic field coupling to overhead and buried wires, Engineering analysis with boundary elements. 35, 3; 555-563, 2011. 